\title{
Las actividades en la naturaleza en la formación inicial docente: un acercamiento desde los sentidos
}

Franklin Castillo Retamal

Universidad Católica del Maule

Alejandro Almonacid Fierro

Universidad Autónoma de Chile

\section{Resumen}

El artículo que a continuación se presenta es el resultado de un trabajo de investigación relacionado con el sentido que otorgan los estudiantes de educación física a las actividades en contacto con la naturaleza. En el estudio emerge con nitidez que el currículo escolar del subsector carece de espacios para el desarrollo y despliegue de este núcleo temático. Por otro lado, permite visualizar la pertinencia del modelo curricular de educación física como disciplina, entendiendo que este enfoque paradigmático sobrepasa la perspectiva mecanicista, dualista e instrumental asignada tradicionalmente a este sector de aprendizaje, para centrar su mirada en la resignificación que emerge de las concepciones y vivencias de los sujetos en proceso de formación inicial. En consecuencia, el propósito central es comprender cómo los futuros profesores otorgan sentido y significado a la relación que establecen con el medio natural, toda vez que entienden la generación de un vínculo eminentemente complejo, que configura el ser humano a partir de la biogeoestructura de los espacios naturales, la relación hombre naturaleza y la propia historicidad del sujeto. Por otro lado, esta investigación se enmarca en el paradigma interpretativo y la metodología cualitativa, toda vez que lo central es comprender las nociones de sentido de profesores de educación física en formación, en el marco de las actividades en el medio natural.

\section{Palabras clave}

Sentido - Profesores en formación - Actividades en la naturaleza.

\section{Correspondencia:}

Franklin Castillo Retamal

Facultad de Ciencias de la Educación

Avenida San Miguel, 3605

Talca - Chile

fcastillo@ucm.cl 


\title{
Activities in nature in initial teacher training: an approach focusing on meanings
}

Franklin Castillo Retamal

Universidad Católica del Maule

Alejandro Almonacid Fierro

Universidad Autónoma de Chile

\begin{abstract}
The paper presented as follows is the outcome of an investigation associated with the meaning that physical education students give to the activities in contact with nature. The study clearly reveals that the school curriculum of this subsector lacks room for the development and deployment of this subject. On the other hand, it allows us see the adequacy of the curriculum model of physical education as a discipline, as this paradigmatic approach overcomes the mechanical, dualist and instrumental perspective traditionally assigned to this learning sector and focuses on the re-signification that emerges from the concepts and experiences of the individuals who go through the process of initial education. As a result, the main purpose is to comprehend how future teachers assign sense and meaning to the relationship they establish with the natural environment, since they understand the generation of an eminently complex link, which configures the human being from the viewpoint of the bio-geo-structure of the natural spaces, man/nature relationship and the very historical character of the individual. On the other hand, the landmark of this research is the interpretative paradigm and the qualitative methodology, since the core question is to comprehend the notions of meaning of physical education teacher whose education is in progress, in the perspective of the activities in the natural environment.
\end{abstract}

\section{Keywords}

Meaning - Teachers under training - Activities in nature.

\author{
Contact: \\ Franklin Castillo Retamal \\ Facultad de Ciencias de la Educación \\ Avenida San Miguel, 3605 \\ Talca - Chile \\ fcastillo@ucm.cl
}


El sustento epistemológico de esta investigación sienta sus bases en los sentidos expresados por los profesores de Educación Física en formación de la Universidad Católica del Maule sobre las actividades en contacto con la naturaleza y emerge desde el trasfondo cultural acumulado en los años de escolaridad.

Desde esta perspectiva, la configuración de este sentido entrega los insumos para la interpretación de sus relatos y permite la comprensión de la significación que otorgan a las actividades en contacto con la naturaleza.

$\mathrm{Al}$ hablar de sentido, primeramente habrá que responder a qué se entiende por el mismo. Habitualmente está presente en el lenguaje cotidiano, cuando se intenta dar significado a algún hecho. Para algunos autores la palabra sentido no podría definirse por sí misma, ya que toma vida cuando se entretejen los elementos que la configuran.

Según Holzapfel (2005), el sentido contieneporlo menos tres acepciones principales: significado, justificación y orientación, donde la primera - significado - genera una distinción sobre hechos, cosas o palabras, asignándoles un valor desde el propio sujeto. La justificación intenta argumentar los significados emergentes; mientras que la orientación se encuentra referida a la direccionalidad que se traza al momento de tomar decisiones dentro de la compleja existencia humana. Es posible que estas dimensiones se sustenten en la emoción y la trascendencia, acoplándose de esta manera cinco generadores de sentido: vínculo, cobijo, atadura, reiteración y sostén.

Feitosa (2006), indica que es por medio de los sentidos que se logra llegar a la sensación, de ella a la emoción y de ahí a los sentimientos, para luego sumergirse en la conciencia, de la cual cada individuo precisa en su proceso personal y profesional como fruto de un medio cultural que lo impregna, de una praxis que lo caracteriza y de un estilo que lo distingue como único. A partir de esta perspectiva, se logra comprender cómo los sentidos traspasan las definiciones unidireccionales, referidas, por un lado, a los clásicos conocidos como vista, oído, tacto, gusto y por otro, a los sentidos entendidos desde el significado de existencia, a partir de las propias vivencias. "En fenomenología, la vivencia se entiende como unidad de vivencia y de sentido, es entonces, una unidad de significación" (GALLO, 2010, p. 37). Es una relación dinámica que emerge de la información obtenida a través de órganos sensoriales, por tanto corporales, lo que permite hacer conciente el significado de una persona, de un objeto o de una situación, haciendo distinciones claras al momento de optar o decidir frente a un evento dado. Schütz (1993) plantea que el significado es la manera en que el yo considera su vivencia, por lo tanto podemos concluir que la construcción del sentido es compleja y multidimensional además de ser permanente e ininterrumpida. Para David Le Breton (1999),

el mundo lo vamos trazando desde la simbólica corporal, lo que se entiende como una memoria que hay que mantener y alimentar constantemente en el espejo del comportamiento y las palabras de los otros... (p. 34),

que en definitiva se constituye en una contribución a la construcción del propio sentido en su contexto, en tanto "la naturaleza humana es un ser dado y la cultura pertenece a aquello que el hombre produce o construye con otros" (BABOLIN, 2005, p. 73).

Según algunos planteamientos de OrtizOsés (2003), la clave de toda filosofía es el sentido, ya que con el tema de la existencia se entra en el ámbito de la vivencia y la experiencia profunda. El autor señala que en la posmodernidad ya no se habla de un sentido desde la razón absoluta o fundamentalista. El sentido es anti absolutista por cuanto es considerado relacional y abierto. Esta acotación no debe caer en el relativismo, puesto que hay un sentido profundo en el amor, el arte, la religión, la belleza y en la actitud de hombre o mujer implicada en la creación de mundo. De este modo, el sentido es flotante pero no ligero, no es 
absoluto pero tampoco relativista: el sentido para Ortiz-Osés es relacional o relacionalista y con una proyección que se apoya en la experiencia. Se podría hablar, entonces, del sentido como una ilusión, que al mismo tiempo es constitutiva del hombre, es como una realidad simbólica sin la cual no podría existir.

La elaboración y posterior puesta en marcha de esta investigación responde a una idea central basada, principalmente, en abordar lo que desde la vivencia se puede expresar y es así que para efectos de este trabajo se ha intentado una aproximación al concepto de sentido, que en palabras de Frankl (2000) es una toma de conciencia de la realidad, a partir de las propias vivencias y relaciones.

Así mismo, plantea que el significado de la vida difiere de un hombre a otro, de un día para otro, como así también el sentido. Así pues, lo que importa no es el sentido en términos generales, sino el significado concreto que el individuo le otorga en cada etapa de su vida. No puede encasillarse como algo único, sino que es un conjunto de situaciones, personas y sentimientos que configuran la significación de acuerdo al momento que está viviendo.

Desde aquí emerge, entonces, la pregunta de investigación ¿cuáles son los sentidos que los estudiantes de Educación Física de la Universidad Católica del Maule le otorgan a las actividades en contacto con la naturaleza?

\section{Actividades en la naturaleza}

La naturaleza siempre ha sido un marco privilegiado para el desarrollo de todo tipo de actividades físicas y deportivas; sin embargo, es necesario establecer la diferencia que existe entre poner en práctica actividades que se desarrollan en un gimnasio, piscina, sala o polideportivo y que, esporádicamente son transportadas a un entorno natural y el grupo de actividades que solamente pueden ser desarrolladas en este entorno. Este conjunto de actividades presenta una identidad propia, tal y como sucede con todas las otras actividades y manifestaciones deportivas.
Así, desde un primer momento es importante aclarar que, por ejemplo, plantear a un grupo de niños un juego de pelota en el medio natural tiene un alcance y unas consecuencias muy distintas a lo que supondría iniciarles en canotaje. En efecto en el primer caso, el entorno no influye esencialmente en el desarrollo del juego ni establece grandes diferencias con su práctica en un gimnasio. Sin embargo en el segundo ejemplo, el entorno, en este caso el agua o el viento entre otros muchos factores, va a ser el que determine el tipo de respuesta motriz del niño y, a largo término, su aprendizaje.

Como se aprecia, en este tipo de actividades el carácter cambiante de la naturaleza va a convertirse en el principal exponente de su desarrollo y precisamente va a ser la incertidumbre asociada a sus imprevisibles cambios el principal rasgo que las define y diferencia.

Actualmente este grupo de actividades se conoce como actividades físico-deportivas en la naturaleza y tienen, en líneas generales, como objetivo común el desplazamiento individual o colectivo hacia un fin más o menos próximo utilizando o luchando con los elementos que constituyen el entorno físico (ASCASO, 1996).

Con el término entorno físico, aludimos a la naturaleza en sus formas más variadas. Así podemos citar actividades en apariencia tan dispares como la escalada o el esquí. Ambas tienen lugar en diferentes medios: la roca y la nieve. De igual modo las técnicas $\mathrm{y}$ patrones motrices que exigen son dispares; sin embargo no solo las dos implican un desplazamiento sino que también durante su práctica el niño debe adaptar su conducta a situaciones cambiantes inciertas, debe tomar decisiones en el transcurso mismo de sus acciones; en definitiva va fluctuando entre la colaboración con el medio - agarrarse a un saliente de la roca - y su superación flexionar más las piernas para salvar un bache. El practicante de estas actividades por tanto, ha de ser considerado como un Ser en relación continua con el medio (1996). 
El concepto de actividades en la naturaleza tiene tantas acepciones como autores que han intentado definirlas. Comenzando por la definición y objetivos que nos presenta el Ministerio de Educación de Chile, se entiende el eje curricular de las Actividades Motrices en Contacto con la Naturaleza, como

la redefinición de lo que en el pasado se entendió como la dimensión recreación en las actividades de Educación Física. Sus propósitos formativos amalgaman actividades motrices, la relación con el medio natural y el aprendizaje sobre el riesgo y la aventura. (MINEDUC, 1998, p. 206)

Además, apunta que las Actividades Motrices en Contacto con la Naturaleza y de Aventura son

Actividades motrices que se practican en el entorno natural sin estandarizarlo. Por lo tanto utilizan su biogeoestructura, es decir, sus características ecológicas integradas en la complejidad del paisaje, como elementos de incertidumbre. De esta manera, las respuestas motrices se adaptan a las condiciones definidas por el medio natural no modificado, generando la necesidad de contactarse con él, desde el ámbito de la praxiología motriz. (MINEDUC, 2007, p. 14)

Según Callejón y De Haro (1993, p. 78), "son aquellas actividades que se pueden realizar dentro o fuera de nuestro entorno habitual con un objetivo principal, el educativo".

Para Alonso (1992),

son aquellas actividades que se realizan en el campo bajo un punto de vista educativo y utilitario, ayudados por el desarrollo de actividad física e incluyendo las de aula y taller que nos proporcionen conocimientos y materiales que se puedan aplicar antes o después en el desarrollo de las mismas. (p. 89)
En el caso de Guillén (2000), las actividades físico deportivas en el medio natural

son aquellas actividades que se realizan en colaboración con el medio y que tienen como objetivo común el desplazarse individual y colectivamente hacia un fin más o menos próximo utilizando o luchando con los elementos que constituyen el entorno físico. (p. 13)

Para nuestros efectos y según la dinámica propiciada en nuestro contexto, definiremos las actividades en la naturaleza como actividades que se desarrollan utilizando la biogeoestructura de los entornos naturales en lo específico y comprendiendo la relación indisociable que existe entre el hombre y la naturaleza en términos holísticos (CASTILLO, 2011).

Desde esta descripción y concepción, las actividades en contacto con la naturaleza colaboran en derribar ciertos paradigmas occidentales en los que priman las dicotomías, en este caso Hombre-Naturaleza, bajo los cuales este Ser Humano no alcanza a conocer y reconocerse parte constituyente de la misma, es integrante como componente de la biodiversidad, vale decir, no existe hoy en nuestras sociedades una mirada con conciencia planetaria y a la vez compleja donde el uno es con lo otro y con los otros. Nos relacionamos en el lenguaje, en la comunión con los otros y los sentidos otorgados a las acciones humanas, que es finalmente la humanización de este Ser Humano.

\section{Metodología}

\section{Tipo de investigación}

Para comprender e interpretar los sentidos expresados por los profesores en formación, se acudió a la metodología cualitativa, la que se refiere en su más amplio sentido a la investigación que produce datos descriptivos, vale decir, las propias palabras de las personas, habladas o escritas y la conducta observable. 
Según Taylor y Bodgan (1987), la metodología cualitativa es más que un conjunto de técnicas y procedimientos para recoger datos, más bien es una forma de encarar el mundo empírico.

Para este estudio se utilizó el Paradigma Interpretativo, puesto que posibilita profundizar el conocimiento y comprensión de cómo la vida social se percibe y experimenta tal y como ocurre, permite incorporar la aparición del sujeto, de los actores frente a lo instituido, sustituyendo los ideales, teorías de explicación, predicción y control por las de comprensión, significado y acción.

Lo que se pretende con este tipo de investigación cualitativa-interpretativa es intentar comprender la realidad desde adentro, priorizando lo individual, lo subjetivo y la concepción de la realidad social, limitándose a observar y describir los fenómenos en un ambiente natural, tal y como se presentan en la realidad.

El enfoque utilizado fue el fenomenológico, que se caracteriza por destacar el énfasis sobre lo individual y la experiencia subjetiva. Lo que pretende la fenomenología es conocer los significados que los individuos dan a su experiencia, por lo tanto, es importante aprehender el proceso de interpretación con el que la gente define su mundo y actúa en consecuencia. Por otro lado, el

fenomenólogo intenta ver las cosas desde el punto de vista de otras personas, describiendo, comprendiendo e interpretando. (RODRÍGUEZ, 1996, p. 40)

\section{Técnica e instrumentos}

Para la recogida de datos se utilizó la técnica de grupos focales, que se define como

entrevistas de grupo, donde un moderador guía una entrevista colectiva durante la cual un pequeño grupo de personas discute en torno a las características y las dimensiones del tema propuesto por la discusión. (MELLA, 2003, p. 176)
Los instrumentos utilizados fueron el diario de campo, la grabadora manual y una cámara digital de video.

\section{Criterios de rigor}

Estos criterios de rigor se establecieron de acuerdo a lo planteado por Vasilachis (2006): Análisis de las entrevistas, Credibilidad, Compromiso con el trabajo de campo, Obtención de datos ricos y Triangulación.

\section{Proceso de recolección y análisis de la información}

La población de estudio para esta investigación fueron estudiantes de Pedagogía en Educación Física de la Universidad Católica del Maule, seleccionados al azar e invitados verbalmente a participar en forma voluntaria. Una vez definidos los sujetos, se les cita a una reunión de acuerdo a fechas acordadas en la oportunidad en las dependencias de la Universidad Católica del Maule. La entrevista se desarrolla en dos grupos de diez personas en días y horarios distintos. Como parte de la motivación se transitó a ratos con preguntas elaboradas con anterioridad.

- Se realizan dos reuniones de conversación (una con cada grupo);

- El documento final que se origina es sometido a codificación manual.

Posteriormente se llevaron a cabo las etapas de transcripción, categorización de la información, sistematización, análisis y reducción de datos.

Atendiendo el análisis de las entrevistas y respondiendo a una expectativa metodológica a partir del habla de los sujetos entrevistados, la resultante de la aplicación de la técnica de investigación denominada grupo focal acredita la posibilidad de "actualizar una estructura y clima social a partir de las interacciones entre los individuos" (MELLA, 2003, p. 214). Esto posibilita configurar criterios que permiten sustentar lo revelado por los jóvenes investigados, favoreciendo 
la profundidad y comprensión del fenómeno estudiado. El producto obtenido de los grupos focales se utiliza, entre otros, para examinar las distancias que existen entre los sujetos, las relacionalidades, el saber cómo otros perciben la realidad y las posibilidades de entenderse en el lenguaje. Permite también enfrentar la complejidad de las preguntas, escuchar las inquietudes, los desacuerdos $\mathrm{y}$ acuerdos mutuos, en la dirección que toma la conversación hacia tópicos que se desean profundizar. En definitiva, es propicio para entender la diversidad de temas, las percepciones y vivencias experimentadas por los sujetos investigados. El proceso de análisis de datos se realizó con un procedimiento inductivo, utilizando como técnica el análisis de contenido, cuyo primer paso consiste en la codificación de los datos obtenidos, para luego construir la matriz de sistematización.

Las categorías previas surgen al levantar el marco teórico de esta investigación, que situada desde el paradigma interpretativo, orienta la comprensión de los sentidos que los profesores en formación otorgan a las actividades en contacto con la naturaleza, desde la red de significaciones propias de estos sujetos, en un proceso de conocer sus experiencias y vivencias a través del diálogo que se despliega desde el discurso de los propios protagonistas. Según Strauss y Corbin
(2002, p. 36), las categorías son "conceptos que representan fenómenos" y estos a su vez se agrupan en términos de propiedades y dimensiones para su posterior análisis. A continuación, se procedió al levantamiento de las categorías axiales que según esos mismos autores buscan realizar un análisis intensivo sobre una categoría para poner de manifiesto las relaciones entre esa categoría y otras subcategorías.

Así, se logró establecer las categorías y sus descriptores, los que se detallan en los resultados y análisis de los mismos. La presentación de éstos siguen la siguiente lógica: en una primera parte, se entregan las macro categorías y las categorías primarias con su respectivo descriptor y los relatos más significativos de cada una de ellas. Posteriormente en el capítulo de Interpretación de los Resultados, se entregan las reflexiones y principales hallazgos de cada una de ellas.

\section{Resultados}

Tras la codificación y categorización levantada luego del análisis de las entrevistas, los sentidos expresados por los estudiantes de Educación Física de la Universidad Católica del Maule acerca de las actividades motrices en contacto con la naturaleza (AMCN) se pueden clasificar de la siguiente manera:

\section{Matriz de sistematización}

\begin{tabular}{|c|c|c|c|c|}
\hline $\begin{array}{l}\text { Macro } \\
\text { categorías }\end{array}$ & $\begin{array}{l}\text { Vivencias en el medio } \\
\text { natural }\end{array}$ & $\begin{array}{l}\text { Concepción de las actividades } \\
\text { en el medio natural }\end{array}$ & $\begin{array}{l}\text { Caracterización de la } \\
\text { formación inicial }\end{array}$ & $\begin{array}{l}\text { Expectativas de las AMCN } \\
\text { en el contexto escolar }\end{array}$ \\
\hline \multirow{4}{*}{$\begin{array}{l}\text { Categorías } \\
\text { primarias }\end{array}$} & $\begin{array}{l}\text { Actividades en la sesión } \\
\text { de Educación Física }\end{array}$ & Formación integral & Formación pertinente & $\begin{array}{l}\text { Se requiere de profesores } \\
\text { con mayores competencias }\end{array}$ \\
\hline & $\begin{array}{l}\text { Actividades } \\
\text { extraescolares }\end{array}$ & Favorece la interdisciplinariedad & Área con menos atención & $\begin{array}{l}\text { Propiciar el trabajo } \\
\text { multidisciplinar }\end{array}$ \\
\hline & $\begin{array}{l}\text { Ausencia de vivencias en } \\
\text { el medio natural }\end{array}$ & $\begin{array}{l}\text { Espacialidad enriquecedora para } \\
\text { el aprendizaje }\end{array}$ & $\begin{array}{l}\text { Currículum adecuado para } \\
\text { el contexto escolar }\end{array}$ & $\begin{array}{l}\text { Incorporación de padres y } \\
\text { familia }\end{array}$ \\
\hline & & $\begin{array}{l}\text { Instancia para el aprendizaje } \\
\text { significativo }\end{array}$ & $\begin{array}{l}\text { Salidas a terreno } \\
\text { significativas }\end{array}$ & $\begin{array}{l}\text { Superar la educación física } \\
\text { tradicional }\end{array}$ \\
\hline
\end{tabular}




\section{a) Vivencias en el medio natural}

Esta corresponde a una categoría que emerge a partir de los relatos de los entrevistados, rememorando los momentos vividos en relación a las actividades en contacto con la naturaleza en la etapa escolar. Alcanza relevancia por cuanto las palabras que describen sus vivencias dan el sustento para finalmente comprender su postura respecto al tema en cuestión, además de caracterizar a grandes rasgos el desarrollo e implementación de este eje curricular en la escuela del siglo XXI.

En el contexto de la macro categoría y mediante las significaciones construidas por los sujetos investigados, se generaron tres categorías primarias: Actividades en la sesión de Educación Física, Actividades extraescolares y Ausencia de vivencias en el medio natural. Estas se conformaron a través de las experiencias que los sujetos investigados han vivenciado y no vivenciado durante su etapa escolar.

Los estudiantes expresaron que sus clases de Educación Física eran monótonas y aburridas, también que existe una exigencia y presión psicológica al momento de realizar actividades. Dieron a entender que la clase de Educación Física en su contexto escolar está orientada y condicionada, aparentemente, a la práctica y entrenamiento de la condición física y del deporte.

Siempre se enfocaba al básquetbol, a las áreas básicas y el tercer eje se dejaba como de lado en el colegio. (P. $1^{1}$ )

Los profesores de Educación Física generalmente se dedicaban como a los deportes tradicionales, individuales $\mathrm{y}$ colectivos. (P. 2)

Se puede decir entonces que las clases de Educación Física se transforman en un

1- Las entrevistas grupales fueron compiladas en un solo texto y numerados los párrafos, de tal manera de especificar la disposición narrativa de los testimonios, vale decir el código $P$, corresponde al párrafo y el numeral, a la ubicación del párrafo en el texto. momento que no propicia mayores intenciones que el adiestramiento y la repetición y se configuran en un siempre lo mismo (JARAMILLO; QUILIND0, 2006).

También se desprende de esta macro categoría que el eje de los juegos deportivos y las actividades de expresión motriz son la base de la sesión de Educación Física en la escuela y no se deja espacio ni oportunidad de acercamiento a las otras áreas de desarrollo. El eje Actividades Motrices en Contacto con la Naturaleza, a menos que exista alguna inquietud del profesor por abordarlo, se implementa por cumplir con la actividad, pero no de forma seria y adecuada.

En media y básica era puro deporte colectivo, deporte individual y nada más que eso. Los profesores ni siquiera conocían esta área, no se manejaban. A lo más lo que era el ámbito del scout pero ni siquiera eso se trabajaba dentro del colegio. (P. 3)

De igual forma, se establece que las vivencias en el medio natural en la etapa escolar para los sujetos involucrados en este estudio, no pasaron de ser situaciones puntuales y no siempre en contexto de aprendizaje formal.

En mi caso yo tuve en esa etapa las salidas de scout, pero ya refiriéndose al colegio, de tercero a cuarto medio el profesor de Matemática, que era nuestro profesor jefe, nos llevaba a acampar y él nos pedía que hiciéramos una serie como de rutinas y que planificáramos el campamento y todo lo que tenía que ver con ello. (P. 7)

Bueno, dentro de las experiencias que tuve yo en educación media, fueron aproximadamente tres, pero también viene por un cuento que no viene del profesor de Educación Física, sino que viene por un profesor de Artes Plásticas y el era... dentro del liceo en el que estudiaba yo, formó un grupo de forjadores ambientales, entonces él se preocupaba de a nosotros sacarnos a campamento. (P. 8) 
Sumado a ello, se encuentra también que, a pesar de que en los planes y programas de estudio está explícitamente expuesto el desarrollo del eje y en porcentajes no menores, los estudiantes manifiestan la total ausencia de estas actividades en sus establecimientos educacionales

Yo no tuve ninguna experiencia en el ámbito escolar de las actividades en contacto con la naturaleza. (P.10)

Bueno, en mi caso, tampoco tuve salidas a terreno, nunca. Ni en la enseñanza media ni básica y una vez le planteamos el tema al profesor y dijo que él no estaba capacitado para andar con tanta gente en zonas abiertas o cosas así. (P.11)

Bueno, ahondando también un poco en la primera pregunta, yo en mi etapa escolar no tuve ningún acercamiento en contacto con la naturaleza, es más, siempre fue, trabajamos con profesores que son, son de la antigua digamos, que nos pasaban una pelota de fútbol y nos mantenían jugando toda la hora de clase. (P.15)

El desarrollar la Educación Física en la escuela de la manera como se ha planteado anteriormente, deportivizada, poco ordenada o dedicada al juego, no sólo es parte de las prácticas de los propios de la especialidad, sino que se ha transformado en el referente indiscutido para el resto de los profesores que hacen las clases de Educación Física sin ser del área, pero que por motivos administrativos han debido hacerse cargo de las mismas y, por lo tanto, no se les puede exigir mejora o innovación si lo que replican es precisamente lo que observan.

De acuerdo a lo manifestado por los sujetos de esta investigación, se puede inferir que las vivencias en el medio natural en la etapa escolar se han visto más bien restringidas por cuanto las posibilidades de acción entregadas por los espacios escolares se limitan al uso de elementos configurados para la práctica de los deportes y no entregan oportunidad para la participación y desarrollo de otras áreas pertenecientes a la disciplina.

\section{b) Concepción de las actividades en el medio natural}

Esta categoría surge desde el problema de la investigación. Es la base de los sentidos que los entrevistados tienen en relación a las actividades en el medio natural, en otras palabras, logra fundamentar la concepción que poseen de esta área los profesores en formación.

Mediante los sentidos que emergieron a partir de los relatos de los sujetos investigados, se puede interpretar y comprender que la concepción de las actividades en el medio natural, compuesta por las categorías primarias: Formación integral, Favorece la interdisciplinariedad, Espacialidad enriquecedora para el aprendizaje e Instancia para el aprendizaje significativo, donde los sujetos, expresando su punto de vista, generaron representaciones de las actividades en el medio natural. A partir de sus relatos, se puede desprender que valoran mucho el hecho que las actividades en la naturaleza entreguen espacios y momentos en los cuales se puedan expresar y manifestar de acuerdo a sus estados de ánimo $\mathrm{y}$, al mismo tiempo, compartir las emociones que afloran. Así mismo, aparecen conductas que habitualmente no surgen en un estado distinto (momento-espacio-sentido), que dan cuenta de los valores educativos que encierra.

Por ejemplo lo transversal se trabaja mejor yo creo en estas actividades, lo que es trabajo de confianza, lo que es confiar en el compañero en el sentido de mi seguridad. (P.16)

Yo creo que es importante... o sea, donde se ve el significado de las actividades en contacto con la naturaleza es que entrega, o sea, refuerza mucho más lo transversal. (P.17) Involucra lo transversal, los valores y se trabaja mucho la solidaridad. (P.19)

Los diferentes sentidos de los sujetos investigados permite concluir que reconocen en 
estas actividades una instancia de crecimiento que no se da en otros ambientes y lugares, situación que sugieren los planes y programas de estudio del Ministerio de Educación (1998, 1999, 2007), al plantear que se deben enfocar las actividades del sector de manera que ayuden a los alumnos y alumnas al conocimiento y confianza de sí mismos, de sus potencialidades y limitaciones, así como en la consolidación de su autoestima y sentido positivo ante la vida. Sin duda que el reforzamiento del ámbito actitudinal se ve ampliamente favorecido en estas actividades por sobre lo procedimental y conceptual, encontrando de cierta manera un equilibrio en el desarrollo del sub sector.

En las palabras de los profesores en formación, afloran mensajes para integrar nuevos aprendizajes en las sesiones de Educación Física y frente a esta necesidad, se concluye que generar otras y nuevas instancias de aprendizaje muestra el espacio para la reflexión que se debe realizar sobre los procesos y las actividades realizadas.

Y aparte que se complementa con otros ramos, como decía mi compañero, no es solamente Educación Física, se trabaja el cuerpo, es verdad y las cualidades que supuestamente trabajamos nosotros todos, pero también involucramos otros como la historia, entonces se complementa mucho más con otros ramos que Educación Física solamente. (P.32)

Bueno, en ese sentido yo creo que más que pertinente, es importante. Es un trabajo interdisciplinar en que se realiza y se trabaja en conjunto con otras disciplinas porque las actividades en contacto con la naturaleza no solo puede trabajar el área de Educación Física sino que la biología, la historia, lenguaje, artes... todas las áreas en conjunto... entonces eso al alumno lo hace crecer mucho más como persona y valorarse también. (P.33)

Así mismo, los sentidos que otorgan los sujetos investigados evidencia que este tipo de encuentro-aprendizaje va más allá de la sola instrucción, va al encuentro desde el relato, de las potencialidades humanas en toda su unidad y sobrepasa lo que puede hacer en función de su bagaje cultural, edad o condición social. Se supone que cada persona aprenderá a partir de su propia conciencia en el espacio-tiempo además de sus propias acciones al momento de aprovechar sus posibilidades para sentir, vivir y actuar usando el pensamiento autónomo, la libertad responsable y el liderazgo propio. A partir de ello, el participante de estas actividades no se transforma en un espectador sino más bien en el protagonista de su propia educación. Desde allí, la relevancia de estas actividades en los procesos educativos tendientes a la formación integral de la persona, que conozca las partes y comprenda el todo y viceversa.

Es importante, o sea, habiendo lugares que son libres por ejemplo y naturales, por qué voy a estar encerrado haciendo deporte si lo puedo hacer observando la flora y fauna que nos rodea... y quizás es menos estresante estar con cuarenta compadres pedaleando al mismo tiempo que estar con dos o tres pedaleando en un cerro por ejemplo. Es distinto (P. 37)

Yo creo que es enseñar a través de las emociones, porque generalmente cuando uno tiene una salida o no sé, por ejemplo cuando uno está chico y va a algún lugar las cosas que más uno se recuerda son las que te causaron más impacto o emoción, no sé, por ejemplo 'iTe acordai del día en que te caíste y te sacaste la mugre!', es ahí donde empiezas a recordar todo lo que pasó en esa situación, en cambio por ejemplo si estás en una sala y te están enseñando tal cosa, estás como siempre lo mismo, no te emociona, en cambio en la naturaleza es como la madre de las emociones. (P. 38)

Esta categoría entrega la visión de la concepción acerca de las actividades en el medio natural de los sujetos investigados la que, como plantea Decroly (apud RETAMAL, 1998), 
ir al medio natural y humano, es donde en verdad se aprende. En contacto con la naturaleza, aún sin ayuda y dirección del maestro, se aprenden continuas e imborrables lecciones. (p. 3)

El aprendizaje, a través de estas actividades, es mucho más significativo para los alumnos, vivenciar las cosas para uno es mucho más significativo que solamente estar pasando una materia. (P. 39)

Sin duda, el carácter interdisciplinar y la característica integradora en el proceso educativo a partir de estas actividades, son las conclusiones que han sustentado el levantamiento de esta categoría que permite vislumbrar a grandes rasgos y a partir de la invocación emotiva de la vivencia, la Concepción de la Actividades en la Naturaleza que tienen los sujetos investigados.

\section{c) Caracterización de la formación inicial}

Esta categoría presenta la mirada particular de los entrevistados respecto de la formación de profesores de Educación Física, desde el interior, desde el mismo proceso. A su vez, entrega antecedentes relativos al desempeño de los profesores en ejercicio en cuanto al desarrollo del eje curricular en estudio.

Yo creo que, quizás, sí existe que se está impartiendo bien, pero no saca nada alguien solo que esté trabajando en eso porque quizás necesita apoyo y eso es lo que yo creo que falta acá, que se le da mucho apoyo a los deportes colectivos, a todos los demás ramos pero a esto, que es demasiado bueno para la formación integral de la persona, no lo toman mucho en cuenta los profesores. Quizás por una cuestión que viene de antaño, de que están educados en otro tipo de escuela pero yo creo que tienen que abrir un poco más allá sus cabezas y ver de que esto no es que esté de moda sino que es una cuestión que de verdad va a ayudar mucho más a un niño a que sea, o crezca como persona porque al final no sacamos nada con hacer Educación Física a un niñito como que le importe tener un músculo más grande, más grande... ¿qué sacamos con eso? No, yo creo que si lo enfocan y apoyan y gestionan que hayan muchos proyectos que se basen en esto, estaría bien. (P. 48)

Los sujetos investigados, a la luz de sus narraciones, estiman que la formación en esta área es pertinente al curriculum y la consideran importante por las características que posee y procura una formación integral, en tanto aborda distintos ámbitos y áreas de conocimiento.

Así mismo, se puede observar que los sujetos investigados afirman haber recibido una formación pertinente y coherente en relación a las actividades en contacto con la naturaleza, contando con herramientas que le permitirán desenvolverse en el ámbito laboral de manera eficiente y responsable.

Yo creo que es demasiado pertinente hacer estas actividades. Los estudios que se han realizado dicen que un niño mientras a más temprana edad se le comience a implantar la actividad física o la expresión corporal, mientras más pequeños es mayor el desarrollo como persona, el desarrollo sicológico que va a tener y más aún va a ser si nosotros estamos orientándolos a que cuide la naturaleza, a tener aprendizajes significativos en cuanto a sacarlos de un ambiente en el que generalmente no se vive y son aprendizajes significativos que van a tener. (P. 50)

$\mathrm{Al}$ mismo tiempo, entregan importante información acerca de las características de las actividades en terreno, que sobrepasan lo habitualmente conocido y se aleja del concepto de paseo con el cual comúnmente se asocia la acción pedagógica en los espacios naturales. 
Yo quería agregar otro dato más, que las actividades motrices en contacto con la naturaleza, aparte que como dijo mi compañero está el ámbito físico, el ámbito afectivo, como en todo rango de aprendizaje, como nosotros hemos aprendido en todo el tiempo que llevamos en la Universidad también hay un ámbito cognitivo, que también vivimos en nuestra salida a terreno, entonces más bien que clasificarlo deberiamos todo unirlo en un solo punto para lograr los aprendizajes que, desde mi punto de vista, son mucho más significativos en esas salidas que estar en una sala escribiendo, pasando materia y aprendiéndose cosas de memoria. (P. 51)

Se puede observar que ha comenzado un cambio en la forma de abordar esta área en la formación inicial, lo cual repercutirá en los procesos llevados a cabo en el aula. Se vislumbra un acercamiento, finalmente, al desarrollo de esta área que ha mantenido su arranque en las intenciones de quienes creen en una forma distinta de abordar y propiciar los procesos de aprendizaje.

Esta mudanza, no sucede por acaso ni de repente. La construcción del conocimiento es la tarea noble de la vida y necesita de tiempo y calma. Porque el corte epistemológico, que marca el proceso evolutivo del conocimiento, es un largo y complejo movimiento que se revela súbito como un relámpago, pero que fue preparado en la oscuridad y en el misterio: como la flor es el trabajo de la semilla en la profundidad de la tierra (FEITOSA, 2006).

\section{d) Expectativas de las AMCN en el contexto escolar}

Esta categoría hace referencia a las posibilidades de acción de los profesores una vez en el sistema escolar, teniendo en cuenta su propia formación y la historicidad de las prácticas de Educación Física en aquellos espacios.

En este sentido, se vislumbra una realidad que aparentemente atenta contra el desarrollo del eje y tiene relación con los propios encargados de llevar a cabo las actividades. Proponen que la formación recibida anteriormente a este periodo no se ajusta a las necesidades curriculares de hoy.

Yo creo que el principal factor es la ignorancia, porque antes de ver esto no lo conocíamos. Los profesores antiguos no lo conocen, no conocen lo rico que es trabajar en contacto con la naturaleza. Yo creo que ese es uno de los principales factores que imposibilita trabajarlo o que no se ha trabajado. (P. 57)

Bueno, la formación docente en este ámbito ha sido débil, absolutamente muy débil e ineficiente, más aún estando dentro de los ejes curriculares del subsector de Educación Física, como si un profesor de cualquier otra asignatura o cualquier otro subsector tuviera en los planes y programas algún contenido y no tuviera idea de ese contenido. No puede pasar eso con nosotros. (P. 58)

De igual forma, los sentidos que expresan destacan y manifiestan que las actividades en contacto con la naturaleza entregan la posibilidad de desarrollar la Educación Física fuera de lo tradicionalmente realizado, vale decir, en espacios desestandarizados que permitan a los estudiantes conocer y re-conocer lugares que son propicios para los procesos de aprendizaje además de incorporar elementos de otras áreas del conocimiento.

Yo creo que si nosotros llevamos la educación propiamente tal a la naturaleza, se ve de otra forma, porque yo tuve una experiencia cuando chico cuando iba en sexto básico que se llamaba escuela al aire libre que pescaban los contenidos de, no sé, de ciencias, matemáticas, castellano y nos enseñaban en una caminata al cerro. Ya la parte de ciencia, cuando estábamos conociendo la naturaleza, ya fue distinto. Conocer matemáticas, empezar a contar, fue distinto. (P. 60) 
Así mismo, los sujetos asignan gran valor a las experiencias obtenidas de las vivencias en el medio natural para su práctica pedagógica, incorporando así características de integración, complemento, emocionalidad, trascendencia y proyección. También la posibilidad de entregar espacios para el desarrollo de la creatividad y favorecer la motivación de los estudiantes, que superaría, en este caso, a lo tradicionalmente observado en las aulas de Educación Física.

Sobre el hecho de sacar a los niños en la clase de Educación Física cambia totalmente el chip de los alumnos y por ejemplo, llevarlos a una actividad en contacto con la naturaleza es fortalecer otro ámbito más del estudiante. (P. 62)

Por ejemplo, ya toda la experiencia que nosotros hemos vivido y en todas las riquezas naturales que Chile tiene, en el ámbito de las actividades en contacto con la naturaleza se podrían desarrollar mucho más en lo que es la educación escolar. (P. 64)

Pareciera ser que los sujetos asignan gran valor a las experiencias obtenidas de las vivencias en el medio natural para su práctica pedagógica, lo que permitiría dar otro cariz a las sesiones de Educación Física en la escuela. Este planteamiento puede conectarse con la Motricidad Humana en el sentido de entregar un momento, traducido en sesión de Educación Física, para presentar actividades con sentido, integradas e integradoras, motivadoras y que den cuenta de una nueva mirada al proceso educativo, como plantea Feitosa (2006), comprometerse con una educación libertadora, que siente sus bases en la creatividad y a partir de ello contribuir al autodescubrimiento del hombre y la construcción de un nuevo mundo, en otras palabras, encontrar una perspectiva renovada del proceso que permita ir proporcionando los medios de aprendizaje, organización y el descubrimiento de esta realidad multidimensional y compleja.

\section{A modo de cierre}

En relación a las cuatro macro categorías, se puede entregar una visión general. En la primera macro categoría y de acuerdo a las historias entregadas y compartidas por los sujetos participantes de esta investigación, podemos inferir que las vivencias en el medio natural en la etapa escolar se han visto restringidas por los espacios escolares limitados al uso de elementos configurados para la práctica de los deportes (materiales y escenarios). Es el entorno el que le dice al estudiante lo que va a encontrar en clase: paisaje no natural, encerrado por muros o mallas que rodean la institución escolar o colegio. Sin embargo, a partir de los planteamientos procedentes del MINEDUC, se observa un esfuerzo, en ningún caso en vano, por tratar de configurar una estructura eficiente y eficaz para el vertiginoso avance de las sociedades modernas, que compromete por cierto, la propia Educación Física y su desarrollo. La escuela de hoy requiere de acciones que denoten y entreguen los espacios para la creación, la emoción y la integración, por lo cual debiera sentar nuevas bases epistemológicas para construir y gestionar un conocimiento que no es absoluto, por el contrario, está en permanente proceso de transformación. Por lo tanto y según lo encontrado en las narraciones revisadas, se debieran adecuar los modelos de enseñanza de tal forma que tiendan al desarrollo de competencias en una espacialidad que salga de los escenarios tradicionalmente utilizados.

A partir de los sentidos de los sujetos investigados, en la segunda macro categoría de la concepción de las actividades en el medio natural, se puede concluir que el hecho de salir del estereotipo de clase o de la estructura en donde se producen los aprendizajes, resulta menos limitante y estresante para quienes participan de aquel proceso y sugiere desarrollar las actividades en otro entorno que resulte más llamativo. Además de eso, los sujetos investigados otorgan cierta importancia a las sensaciones emanadas a partir de lo 
que en términos emocionales les provoca. En consecuencia, desde la concepción de las actividades en la naturaleza de los sujetos investigados, se sostiene que la espacialidad es un elemento importante en los procesos de aprendizaje además de ser propicios para la emergencia de emociones que marcarán hitos importantes en la vida de ese ser humano.

Dentro de la tercera macro categoría, caracterización de la formación inicial, se pude visualizar que los sujetos investigados, a la luz de sus narraciones, estiman que la formación en esta área es pertinente al curriculum y la consideran importante por la particularidad que posee. También procura una formación integral en tanto aborda distintos ámbitos y áreas de conocimiento. A la luz del relato, se puede observar que los sujetos investigados afirman haber recibido una formación pertinente $\mathrm{y}$ coherente en relación a las actividades en contacto con la naturaleza, en la cual las herramientas otorgadas le permitirán desenvolverse en el ámbito laboral de manera eficiente y responsable.

Por último, en la cuarta macro categoría de expectativas de las AMCN en el contexto escolar se puede identificar, a partir de los sentidos de los sujetos investigados, que existe preocupación por la formación inicial y por las acciones que están llevando a cabo los profesionales de Educación Física en ejercicio. Además, manifiestan que es necesario contar con profesores con las competencias necesarias para el desarrollo de las actividades en contacto con la naturaleza en el contexto escolar.

A partir de la visión general de esta investigación y de las opiniones de los sujetos participantes de la misma, permiten estructurar los componentes relativos a las significaciones que los estudiantes de Educación Física de la Universidad Católica del Maule tienen acerca de las actividades en contacto con la naturaleza.

Los aportes que presenta el estudio se centran en posicionar la temática de las significaciones y sentidos como una línea de investigación dentro del campo educativo y a su vez, colaborar al levantamiento de la Motricidad Humana como una Ciencia.

-En consecuencia, este estudio permite acercarse desde los sentidos expresados acerca de las actividades en contacto con la naturaleza a una nueva forma de pensar educativo, en el que las barreras de la historia y la cultura social podrán ser derribadas a partir de la Motricidad Humana como una ciencia que evoca y provoca el enlace epistémico de unión de saberes.

\section{Referencias}

ASCASO, Jorge. Actividades en la naturaleza. Ministerio de Educación y Ciencias. Madrid: Edelvives, 1996.

ALONSO, Víctor. Las actividades en la naturaleza. Manual de monitor. Madrid: Penthatlon, 1992.

BABOLIN, Sante. Producción de sentido. Bogotá: San Pablo, 2005.

CALLEJÓN, Juan; DE HARO, María. Programación de actividades en el medio natural. Madrid: Penthatlon, 1993.

CASTILLO, Franklin. Las actividades en la naturaleza en la formación de profesores. Saarbrücken: Editorial Académica Española, 2011.

FEITOSA, Ana. Mudanzas: horizontes desde la Motricidad. Popayán: Universidad del Cauca, 2006. 
FRANKL, Víctor. En el principio era el sentido, reflexiones en torno al ser humano. Buenos Aires: Paidós, 2000.

GALLO, Luz. Los discursos de la Educación Física contemporánea. Bogotá: Kinesis, 2010.

GUILLÉN, Roberto. Actividades en la naturaleza. Madrid: INDE, 2000.

HOLZAPFEL, Cristóbal. A la búsqueda del sentido. Santiago: Sudamericana, 2005.

JARAMILLO, Luis; QUILINDO, Víctor. Paisajes, contenidos y métodos: sentidos que configuran la Educación Física como un 'siempre lo mismo'. Revista Pensamiento Educativo, Santiago. Ediciones Universidad Católica de Chile, v.38, p. 154-171, 2006.

LE BRETON, David. Las pasiones ordinarias. Antropología de las emociones. Buenos Aires: Nueva visión, 1999.

MELLA, Orlando. Metodología cualitativa en ciencias sociales y educación. Santiago: Grafic Andes, 2003.

MINISTERIO de Educación de Chile (MINEDUC). Decreto Supremo de Educación 220. Santiago: Ministerio de Educación de Chile, 1998.

. Decreto Supremo de Educación 232. Santiago: Ministerio de Educación de Chile, 1999.

. Un análisis sobre la propuesta curricular AMCN y sobre el proceso de implementación curricular en el eje. Periodo 2002-2007. Santiago: Ministerio de Educación de Chile, 2007.

ORTIZ-OSÉS, Andrés. Amor y sentido, una hermenéutica simbólica. Barcelona: Anthropos, 2003.

RETAMAL, Orlando. Una educación para reconciliar al hombre con la tierra. Solo la educación holística hará posible la continuidad de la vida. Revista Estudios Pedagógicos, Valdivia. Universidad Austral de Chile, v. 24, p.107-121, 1998.

RODRIGUEZ Gregorio; GIL, Javier; GARCIA, Eduardo. Metodología de la Investigación Cualitativa. Málaga: Aljibe, 1996.

STRAUSS, Anselm; CORBIN, Juliet. Bases de la investigación cualitativa: técnicas y procedimientos para desarrollar la teoría fundamentada. Medellín: Universidad de Antioquia. 2002.

SCHÜTZ, Alfred. La construcción significativa del mundo social. Barcelona: Paidós, 1993.

TAYLOR, Steve; BODGAN, Robert. Introducción a los métodos cualitativos de investigación. Barcelona: Paidós, 1987.

VASILACHIS, Irene. Estrategias de Investigación Cualitativa. Barcelona: Gedisa, 2006.

Recebido en: 25.07.2011

Aprobado en: 12.03.2012

Franklin Castillo Retamal es académico en la Facultad de Ciencias de la Educación, del Departamento de Ciencias de la Actividad Física de la Universidad Católica del Maule, Talca, Chile.

Alejandro Almonacid Fierro es docente en el curso de Pedagogía en Educación Física de la Facultad de Educación, en la Universidad Autónoma de Chile, sede Talca. Email: aalmonacidf@uautonoma.cl. 
